\title{
A Glance at the Acupuncture Point from the Perspective of Current Technology
}

\author{
Mehmet Tuğrul Cabığlu \\ Department of Physiology, Başkent University Faculty of Medicine, Ankara, Turkey \\ Email: tugcab@gmail.com
}

Received 6 March 2016; accepted 20 March 2016; published 23 March 2016

Copyright (C) 2016 by author and OALib.

This work is licensed under the Creative Commons Attribution International License (CC BY). http://creativecommons.org/licenses/by/4.0/

(C) (i) Open Access

\begin{abstract}
Acupuncture point differs from non-acupuncture points in terms of receptor intensity, primarily noci receptors. This histological difference characterizes the acupuncture points. Among these differences, especially electrical properties of acupuncture points are noteworthy; these points are characterized by increased conductance, reduced impedance and resistance, increased capacitance and elevated electrical potential. The alterations in surrounding tissues and related organs lead to changes in the electrical properties of acupuncture points. I suggest that the changes in the surrounding tissues and related organs can be determined by electrical measurements from acupuncture points. We are in the opinion that electrical changes at acupuncture point may be useful in early diagnosis and treatment by determining the pathological condition of the related organ and around the acupuncture point at the initial stage.
\end{abstract}

\section{Keywords}

Acupuncture Point, Electrical Potential, Impedance, Conductance, Resistance

Subject Areas: Internal Medicine, Psychiatry \& Psychology

\section{Introduction}

The history of acupuncture goes back to at least 3000 years although it differs in different sources [1]. With the advanced technology of today, technological devices that can be used in the diagnosis and treatment of diseases, by particularly measuring the electrical changes in acupuncture points, are designed to be developed.

Acupuncture is classically a treatment method implemented by the insertion of steel, silver and gold needles at acupuncture points. In this treatment method, the characteristics of the acupuncture points are important. All the classic acupuncture sources report that acupuncture points are used in case of organ discomfort in the surrounding tissues and points [2].

According to Traditional Chinese Medicine, acupuncture point is associated with meridians and according to 
neurophysiological approach it is associated with dermatomes related to an organ and cellular connections in the surrounding tissues [3] [4]. It is thought that the alterations in these tissues and organs particularly cause electrical changes in the related acupuncture points.

\section{The Use of Acupuncture Points in the Treatment and Diagnosis of Disease}

According to classical acupuncture theories, acupuncture point is not only used for treatment but also for the diagnosis of diseases. These theories suggest that pain or discoloration at these acupuncture points by exerting a certain level of pressure shows that there may be a problem in the related organ and surrounding tissues [2]. Today, the measurement of electrical and other changes at the acupuncture point may contribute to the diagnosis and follow-up of diseases gain importance.

In case of visceral organ malfunction, positive reactions such as sensitivity or tenderness are found at the corresponding Back-Shu points [5]. In case of dysfunction of the visceral organs, there is pain and sensitivity at the Back-Shu points related to these organs [5] [6]. Segmental dispersion of the sympathetic and parasympathetic systems is related to the location of Back-Shu points. The alterations seen in the visceral organs by applying acupuncture can be attributed to the modulation of sympathetic and parasympathetic responses [3] [7]. In this neurophysiological approach, it is thought that the changes in the visceral organs will lead not only to pain and sensitivity at the related Back-Shu and Front-Mu points, but also will evoke electrical changes.

\section{Histological Characteristics of Acupuncture Points}

The distribution of somatosensory receptors and the number of free nerve endings are different in the acupuncture points. They are also the dense loci for nociceptors, Golgi-tendon receptors, Meissner corpuscles, Krause's end-bulbs and glomus-bodies [8]. In the recent studies, the identification of surface opioid receptors, gammaaminobutyric acid (GABA), bradykinin, histamine, serotonin and capsaicin receptors on nociceptive afferents [9] suggest that pain suppression descended from the spinal cord dorsal horn to nociceptor level.

\section{Electrical Characteristics of Acupuncture Points}

I have mentioned that acupuncture points have different electrical properties. These properties are increased conductance, reduced impedance and resistance, increased capacitance and elevated electrical potential. By utilizing these properties, several devices have been developed for the analysis of acupuncture points for the purpose of identifying acupuncture points and diagnosis. Skin impedance is the resistance of the skin to electrical current flow. Electrical impedance requires two components, resistance and reactance, and reactance is composed of inductance and capacitance [10].

In Traditional Chinese Medicine, it is thought that energy flows through meridians and the acupuncture points are on these meridians. Ahn et al. performed measurements over the pericardium meridian and compared it with the tissues near the meridian, and found that the impedance on the tissues over the meridian was lower; however they found no difference in terms of impedance on the Spleen meridian [11].

The presence of acupuncture points was attempted to be explained by the alterations in skin impedance, temperature and bio fluid dynamics at acupuncture points [12].

\section{Studies Related to the Impedance of Acupuncture Points}

Although Pearson et al. [13] found no significant decrease in the electrical skin impedance of right Yangbai (GB14), right Laogong (PC8) and left Guanchong (TE1) points in the comparison of non-meridian points with points near the meridian in 20 healthy adults, multiple studies found a decrease in impedance [14] [15].

The skin tissue is normal, but there is decreased skin resistance and high conductance at the acupuncture point Decreased skin impedance has been found in the measurements performed at Dingchuan point and along the acupuncture meridian in asthmatic patients, when compared with that of healthy individuals [14] [15]. It was demonstrated that skin conductance was lower along 12 meridians in renal colic patients in comparison to that of the control group [16]. Hsu et al. [17] used heart rate variability (HRV), pulse rate variability (PRV) and skin conductance (SC) of acupuncture points on heart meridian to study the effects of electrical acupuncture (EA) on Xinshu (UB 15) point. HRV, PRV and SC of UB15 point was recorded before and after applying $2 \mathrm{~Hz}$ frequency EA, and compared. This study demonstrated a significant increase in the normalized high frequency power 
component of HRV and PRV, and a significant decrease in the normalized low frequency power when EA was applied to UB15 point. It has been found in several studies that SC of acupuncture points decrease after applying EA.

The alterations in the electrical skin resistance (ESR) at acupuncture points may reflect the physiological and pathological conditions in the human body [18]-[20]. Comparative studies of acupuncture points in healthy individuals and patients may explain the electrodermal properties of acupuncture points. Some studies showed a correlation between the changes in ESR at specific acupuncture points and disease conditions [21] [22].

She et al. [18], performed bilateral ESR measurements at 10 pre-determined acupuncture points in 48 healthy volunteers and 46 patients with primary dysmenorrhea on the first day of menstruation and at the third day after menstruation. While the ESR measurements of the two groups were similar, it was found that ESR measurements of primary dysmenorrhea patients were either higher or lower during menstruation, showing that menstruation changes the ESR of primary dysmenorrhea patients.

From the 10 acupuncture points studied in this study, there was a significant change in the imbalance ratio at Sp6, GB39 and Sp8, which are the points that are frequently used for dysmenorrhea in clinical applications [18] [19]. Some devices have been developed by utilizing the ESR changes at the acupuncture point. Among these devices, mainly those that identify the location of the acupuncture point gain importance, followed by those that are used in the diagnosis of diseases and those developed for research [23].

It is thought that pathological process will evoke more sensitive reactions at the acupuncture points in the tissues where the process develops. A recent study defined the state of acupuncture points as sensitized or awake and rest state [24] [25]. Numerous studies on healthy individuals found no change in the ESR of acupuncture points [11] [26]. It has been stated that the related acupuncture point will pass from "rest status" to "sensitive status” when the body suffers from an illness [27] [28].

Pearson et al. [13], in their study applied direct current (DC) and $100 \mathrm{~Hz}$ alternative current (AC) to three acupuncture points (APs), one site on the related meridian (MP) of each AP and a site off the meridian (NP) of each AP, and compared resistance/impedance at these points. In this study, when GB14 AP was compared with NP and non-acupuncture points, it was found that there was a difference when $100 \mathrm{~Hz}$ AC was applied; however it was seen that electrical impedance decreased at PC8, GB14 and TE1 MPs when compared with non-meridian points, with the exception of the DC resistance at PC8.

The studies mentioned above have suggested that impedance changes in acupuncture points and meridians may be important in the diagnosis and follow-up of diseases [29].

Shan et al. [30] compared the Functional Magnetic Resonance Imaging data they obtained by stimulating real (bilateral LI4 and Liv3) and sham acupuncture points. In this study, in the comparison of real acupuncture points and sham acupuncture points, they found increased neuronal activity in different areas of the brain. They stated that these areas were the superior parietal lobule and postcentralgyrus in the somatosensory cortex, the calcarinegyrus, precuneus, cingulate cortex, and parahippocampalgyrus in the limbic-paralympic system, the fusiform and occipital gyri, basal ganglia, and the cerebellum in the visual-related cortex. This study demonstrated specific activity in the brain by the stimulation of real acupuncture points.

It is mentioned in the present paper that the changes in the surrounding tissues and related organs lead to alterations, especially in the electrical properties of the acupuncture point. I think that electrical measurements from the acupuncture points can allow the determination of the changes in surrounding tissues and related organs. We are in the opinion that electrical changes at acupuncture point may be useful in early diagnosis and treatment by determining the pathological condition of the related organ and around the acupuncture point at the initial stage. In order to accomplish this, multiple detailed studies on the subject is required.

\section{References}

[1] Ullet, G.A., Han, S. and Han, J.S. (1998) Electroacupuncture: Mechanism and Clinical Application. Biological Psychiatry, 44, 129-138. http://dx.doi.org/10.1016/S0006-3223(97)00394-6

[2] Cheng, X.N. (2010) Chinese Acupuncture and Moxibustion. 3rd Edition, Foreign Languages Press, Beijing.

[3] Cabioglu, M.T. and Arslan, G. (2008) Neurophysiologic Basis of Back-Shu and Huatuo-Jiaji Points. The American Journal of Chinese Medicine, 36, 473-479. http://dx.doi.org/10.1142/S0192415X08005916

[4] Cabioglu, M.T. and Surucu, H.S. (2009) Acupuncture and Neurophysiology. Medical Acupuncture, 21, 13-20. http://dx.doi.org/10.1089/acu.2009.0638 
[5] O’Connor, J. and Bensky, D. (1988) An Overview of the Points. In: O’Connor, J. and Bensky, D., Eds., Acupuncture a Comprehensive Text, 1st Edition, Eastland Press, Washington DC, 119-140.

[6] Teitelbaum, D.E. (2000) Osteopathic Vertebral Manipulation and Acupuncture Treatment Using Front Mu and Back Shu Points. Medical Acupuncture, 12, 36-37.

[7] Cabioglu, M.T., Kaya, Y. and Surucu, H.S. (2009) Neurophysiologic Basis of Front-Mu Points. Neuroanatomy, 8, 3235.

[8] Kho, H. and Robertson, E.N. (1997) The Mechanisms of Acupuncture Analgesia: Review and Update. American Journal of Acupuncture, 25, 261-281.

[9] Dray, A. and Perkins, M.N. (1993) Bradykinin and Inflammatory Pain. Trends in Neurosciences, 16, 99-104. http://dx.doi.org/10.1016/0166-2236(93)90133-7

[10] Ahn, A.C. and Martinsen, O.G. (2007) Electrical Characterization of Acupuncture Points: Technical Issues and Challenges. Journal of Alternative and Complementary Medicine, 13, 817-824. http://dx.doi.org/10.1089/acm.2007.7193

[11] Ahn, A.C., Wu, J., Badger, G.J., Hammerschlag, R. and Langevin, H.M. (2005) Electrical Impedance along Connective Tissue Planes Associated with Acupuncture Meridians. BMC Complementary and Alternative Medicine, 5, 10. http://dx.doi.org/10.1186/1472-6882-5-10

[12] Sheu, T.W.H., Huang, V.C. and Rani, H.P. (2008) Development of an Electro-Osmotic Flow Model to Study the Dynamic Behavior in Human Meridian. International Journal for Numerical Methods in Fluids, 56, 739-751. http://dx.doi.org/10.1002/fld.1554

[13] Pearson, S., Colbert, A.P., McNames, J., Baumgartner, M. and Hammerschlag, R. (2007) Electrical Skin Impedance at Acupuncture Points. Journal of Alternative and Complementary Medicine, 13, 409-418. http://dx.doi.org/10.1089/acm.2007.6258

[14] Ngai, S.P. and Jones, A.Y. (2009) Skin Impedance at Acupuncture Point Dingchuan in Subjects with and without Asthma. Chinese Journal of Rehabilitation Medicine, 24, 481-484.

[15] Ngai, S.P., Jones, A.Y. and Cheng, E.K. (2011) Lung Meridian Acupuncture Point Skin Impedance in Asthma and Description of a Mathematical Relationship with Fev1. Respiratory Physiology \& Neurobiology, 179, 187-191. http://dx.doi.org/10.1016/j.resp.2011.08.004

[16] Lee, C.T., Chang, Y.H., Lin, W.Y., Xu, J.M., Chen, H.Y. et al. (2010) Applications of Meridian Electrical Conductance for Renal Colic: A Prospective Study. Journal of Alternative and Complementary Medicine, 16, 861-866. http://dx.doi.org/10.1089/acm.2009.0273

[17] Hsu, C.C., Weng, C.S., Liu, T.S., Tsai, Y.S. and Chang, Y.H. (2006) Effects of Electrical Acupuncture on Acupoint BL15 Evaluated in terms of Heart Rate Variability, Pulse Rate Variability and Skin Conductance Response. The American Journal of Chinese Medicine, 34, 23-36. http://dx.doi.org/10.1142/S0192415X06003606

[18] She, Y.F., Ma, L.X., Qi, C.H., Wang, Y.X., Tang, L., et al. (2014) Do Changes in Electrical Skin Resistance of Acupuncture Points Reflect Menstrual Pain? A Comparative Study in Healthy Volunteers and Primary Dysmenorrhea Patients. Evidence-Based Complementary and Alternative Medicine, 2014, 836026. http://dx.doi.org/10.1155/2014/836026

[19] Royal, F.F. and Royal, D.F. (1991) A Review of the History and Scientific Bases of Electrodiagnosis and Its Relationship to Homeopathy and Acupuncture. American Journal of Acupuncture, 19, 137-152.

[20] Brewitt, B. (1996) Quantitative Analysis of Electrical Skin Conductance in Diagnosis: Historical and Current Views of Bioelectric Medicine. Journal of Naturopathic Medicine, 6, 66-75.

[21] Tsuei, J.J., Lam Jr., F., Mi, M. and Zhao, Z. (1989) Studies in Bioenergetic Correlations—Study on Bioenergy in Diabetes Mellitus Patients. American Journal of Acupuncture, 17, 31-38.

[22] Saku, K., Mukaino, Y., Ying, H. and Arakawa, K. (1993) Characteristics of Reactive Electropermeable Points on the Auricles of Coronary Heart Disease Patients. Clinical Cardiology, 16, 415-419. http://dx.doi.org/10.1002/clc.4960160509

[23] Tsuei, J.J., Lam Jr., F. and Zhao, Z. (1988) Bioenergetic Correlations-Bioenergetic Regulatory Measurement Instruments and Devices. American Journal of Acupuncture, 16, 345-349.

[24] Chen, R.X., Kang, M.F. and Chen, M.R. (2011) Return of Qibo: on Hypothesis of Sensitization State of Acupoints. Zhongguo Zhen Jiu, 31, 134-138.

[25] Cheng, B., Shi, H., Ji, C.F., Li, J.H., Chen, S.L., et al. (2010) Distribution of the Activated Acupoints after Acute Gastric Mucosal Injury in the Rat. Zhen Ci Yan Jiu, 35, 193-197.

[26] Kramer, S., Winterhalter, K., Schober, G., Becker, U., Wiegele, B., et al. (2009) Characteristics of Electrical Skin Resistance at Acupuncture Points in Healthy Humans. Journal of Alternative and Complementary Medicine, 15, 495-500. http://dx.doi.org/10.1089/acm.2008.0331 
[27] Chen, R.X. and Kang, M.F. (2007) Clinical Application of Acupoint Heat-Sensitization. Zhongguo Zhen Jiu, 27, 199202.

[28] Chen, R.X. and Kang, M.F. (2008) Key Point of Moxibustion, Arrival of Qi Produces Curative Effect. Zhongguo Zhen Jiu, 28, 44-46.

[29] Ngai, S.P. and Jones, A.Y. (2013) Changes in Skin Impedance and Heart Rate Variability with Application of AcuTENS to BL 13 (Feishu). Journal of Alternative and Complementary Medicine, 19, 558-563. http://dx.doi.org/10.1089/acm.2012.0097

[30] Shan, Y., Wang, Z.Q., Zhao, Z.L., Zhang, M., Hao, S.L., et al. (2014) An fMRI Study of Neuronal Specificity in Acupuncture: The Multiacupoint Siguan and Its Sham Point. Volume 2014, 1-6. http://dx.doi.org/10.1155/2014/103491 